\title{
Niche diversity predicts personality structure across 115 nations
}

Patrick K. Durkee*1, Aaron W. Lukaszewski², Christopher R. von Rueden ${ }^{3}$, Michael D. Gurven ${ }^{4}$, David M. Buss ${ }^{1}$, \& Elliot M. Tucker-Drob ${ }^{1}$

\author{
${ }^{1}$ The University of Texas at Austin, Department of Psychology \\ ${ }^{2}$ California State University, Fullerton, Department of Psychology \\ ${ }^{3}$ University of Richmond, Jepson School of Leadership Studies \\ ${ }^{4}$ University of California, Santa Barbara, Department of Anthropology
}

*Corresponding author: Patrick Durkee (pdurkee@utexas.edu), The University of Texas at Austin, Department of Psychology, 108 E. Dean Keeton St., Austin, TX 78712. 


\begin{abstract}
The niche diversity hypothesis proposes that personality structure arises from the affordances of unique trait-combinations within a society. It predicts that personality traits will be both more variable and differentiated in populations with more distinct social and ecological niches. Prior tests of this hypothesis in 55 nations suffer from potential confounds associated with differences in the measurement properties of personality scales across groups. Using psychometric methods for the approximation of cross-national measurement invariance, we test the niche diversity hypothesis in a sample of 115 nations $(N=685,089)$. We find that an index of niche diversity was robustly associated with lower inter-trait covariance and greater personality dimensionality across nations but was not consistently related to trait variances. These findings generally bolster the core of the niche diversity hypothesis, demonstrating the contingency of human personality structure on socioecological contexts.
\end{abstract}

Keywords: personality; niche diversity; Big Five; cross-cultural; alignment

\title{
Significance statement
}

We tested the niche diversity hypothesis, which proposes that personality structure arises from the affordances of unique trait-combinations within a society, in a sample of 115 nations. Using methods to ameliorate confounds associated with measuring personality cross-nationally, we found that greater niche diversity robustly predicted less overlap between personality traits but not differences in the variance in personality traits. These findings generally bolster the core of the niche diversity hypothesis, demonstrating the contingency of human personality structure on socioecological factors. 


\section{Niche diversity predicts personality structure across 115 nations}

Humans differ from one another in myriad ways_-physically, behaviorally, and psychologically. Relatively stable patterns of individual differences in patterns of thinking, feeling, and behaving broadly define human personality (McCrae \& Costa, 1990; Briley \& Tucker-Drob, 2014). Inductive methods, such as factor analysis, have characterized person-toperson variability in the broad constellation of personality-relevant traits into five or six major dimensions or factors (Digman, 1990; McCrae \& Costa, 1992; Lee \& Ashton, 2004; Saucier \& Goldberg, 1996). One dominant perspective holds that these factors, and how they vary and covary within populations, result directly from basic biological structures and processes that are intrinsic to the human mind (McCrae \& Costa, 1997; McCrae \& Costa, 2008; Nettle, 2009). According to this universal personality structure view, the same patterns of personality factor variation and covariation will be obtained across populations.

Contrasting with the predictions of the universal personality structure perspective, the number of dimensions needed to summarize personality variation appears to differ somewhat across populations. Whereas differences in personality structure tend to be relatively small across western and industrialized nations (Allik \& McCrae, 2004; Kajonius \& Mac Giolla, 2017; McCrae et al., 1998; Schmitt et al., 2007), inconsistencies are larger when considering low- and middle-income nations (Heine \& Buchtel, 2009; McCrae \& Terracciano, 2005; Saucier et al., 2014). Moreover, research conducted with the Tsimane, a small-scale Amazonian society, suggests that two dimensions - prosociality and industriousness — are sufficient to characterize the major patterns of thinking, feeling, and behaving (Gurven et al., 2013). These Tsimanespecific dimensions each contain heterogeneous mixtures of items from the traditional five-factor 
traits (Gurven et al., 2013), making them qualitatively distinct from other two-factor models observed in industrialized societies (e.g., Digman, 1997).

Differences in personality structure between populations have been presumed by some researchers to stem from measurement artifacts, such as differences in translations or item functioning (Heine \& Buchtel, 2009; McCrae \& Terracciano, 2005). However, with such artifacts controlled, between-population differences in personality structure may also persist as a result of substantive differences in social and cultural dynamics underlying personality development (Briley \& Tucker-Drob, 2017). The niche diversity hypothesis (Lukaszewski et al., 2017; Smaldino et al., 2019) provides one framework for testing such an account.

The niche diversity hypothesis holds that within human populations, individuals occupy different niches: micro-populations within a larger population with different affordances and cost-benefit structures (e.g., organizations, occupations, social and cultural groups, coalitions, families). For example, in human societies, some niches may favor high levels of patience and industriousness with low levels of anxiety, but not specific levels of imaginativeness. Other niches may favor high levels of sociability and risk tolerance, without specifically favoring any level of honesty. And some may not incentivize any particular levels or combinations of traits.

In general, humans tailor behavioral traits to the demands of local socioecological niches (Briley \& Tucker-Drob, 2017; Henrich, 2015; Kaplan et al., 2009; Pinker, 2010; Tooby \& Devore, 1987). The developmental calibration of behavioral profiles may occur through a mixture of social learning (Legare, 2017) and state-behavior feedback loops (Sih et al., 2014). Consistent with the functionality of niche-behavior compatibility, people whose multidimensional personality profiles are a better match with the demands of their niche experience higher material payoffs over time (Denissen et al., 2018). 
According to the niche diversity hypothesis, the number of specialized niches varies widely across human populations. In large complex societies individuals can occupy an assortment of different niches_-each incentivizing different personality-trait optima. In smallerscale societies (e.g., hunter gatherers), the number and diversity of niches that are available to occupy — and the trait levels and combinations required for success within them - tend to be more delimited (Gurven et al., 2013). Thus, the number of niche-incentivized trait profiles is predicted to be greater in some human populations than others (Lukaszewski et al., 2017). Compared to populations with fewer unique niches, populations with more unique niches are hypothesized to exhibit (i) more distinct combinations of traits (i.e., less covariance among traits; Lukaszewski et al., 2017), (ii) wider distributions of trait levels (i.e., more individual variation in each trait; Smaldino et al., 2019), and (iii) more emergent dimensions of personality (Smaldino et al., 2019). Using agent-based models, Smaldino et al., (2019) confirmed that these predicted associations are obtained under conditions in which (a) populations differ in niche diversity and (b) individuals adapt trait levels on the basis of the niches that they occupy.

To date, only one dataset of 55 nations (Schmitt et al., 2007) has been leveraged to empirically test predictions from the niche diversity hypothesis. In this sample, Lukaszewski et al. (2017) found a moderate negative association between nation-level intercorrelations among Big Five traits and a proxy of nation-level niche diversity based on the three indices: Human Development Index, percentage of people living in cities (i.e., urbanization), and the variety of products produced within a nation (i.e., sectoral diversity). Using the same 55-nation sample and niche diversity proxy, Smaldino et al. (2019) found that Big Five trait variance was moderately positively associated with niche diversity. Finally, Del Giudice (2020) applied dimensionality reduction methods to the Big Five covariance estimates provided by Lukaszewski et al. (2017) 
for the 55 nations and found that niche diversity was positively associated with the number of emergent personality dimensions. These studies provide initial support for the niche diversity hypothesis of personality structure.

While these studies implemented controls to assess artifacts of translations and general response tendencies (i.e., acquiescence bias), none has yet implemented rigorous methods to guard against the potential for the focal associations to arise from systematic differences in how the items within personality measures function across populations. The differential functioning of items across populations due to measurement nonequivalence can bias estimates of factor means, covariances, and variances (Byrne et al., 1989; Church et al., 2011). Indeed, Lukaszewski et al. (2017) found that their Big Five measures failed to exhibit the same latent trait structure across nations, thereby potentially invalidating estimates of personality trait variance and covariance derived from factor score composites.

Here, we present a stronger test of test of the niche diversity hypothesis by drawing on new large-scale personality data from 115 nations and a cutting-edge method to approximate measurement invariance for more valid comparison of personality structure across nations. We tested three focal predictions formalized in Smaldino et al. (2019): niche diversity is (i) negatively associated with nation-level covariation among personality factors; (ii) positively associated with nation-level variance across personality factors; and (iii) positively associated with the number of nation-level personality dimensions.

\section{Methods}

\section{Participants}

We drew personality data on 1,015,341 participants from the Open-Source Psychometrics Project (https://openpsychometrics.org/). The datasets from this repository have been used in a 
variety of publications (e.g., Chen, Culpepper, \& Liang, 2020; Hirschfeld, von Brachel, \& Thielsch, 2014; Kaiser, Del Giudice, \& Booth, 2019). The dataset contains responses to personality items along with IP addresses and country of residence; no information on participant sex or other demographics is available. Respondents accessed the survey through the internet and received no compensation for participating. To minimize the potential for multiple instances from the same responders, we removed all participants with identical IP addresses. We also removed participants from nations with fewer than total 90 respondents. After these exclusions, our total sample size for analysis is $N=685,089$ participants across 115 nations.

\section{Measures}

Personality. Personality was measured using the 50-item International Personality Item Pool (IPIP) Five-Factor Markers (Goldberg, 1992), which has strong construct validity (Lim \& Ployhart, 2006) and is generally invariant across gender and American ethnic groups (Ehrhart et al., 2008). Sample items include, "I am the life of the party" (Extraversion), "I am interested in people" (Agreeableness), "I am always prepared" (Conscientiousness), "I am relaxed most of the time" (Emotional Stability), and "I have a rich vocabulary" (Openness). Participants rated the extent to which they agreed that each of the 50 statements was true of them using a five-point scale $(1=$ disagree; $5=$ agree $)$. All instructions and scale items were presented in English to all participants. In our results, we present sensitivity analyses to test the role of English proficiency.

Niche Diversity. We use the same index of niche diversity as Lukaszewski et al. (2017) and Smaldino et al. (2019). A nation's niche diversity is estimated as its score on the first principal component of three nation-level variables: Sectoral Diversity (i.e., the volumeweighted variety of a nation's exports); Urbanization (i.e., percent of the nation's population living in cities); and the Human Development Index (HDI; i.e., a nation's average levels of 
education, gross domestic product, and life expectancy). Each of these three variables is a conceptually appropriate indicator of a nation's niche diversity, given their direct and indirect relationships to economic specialization and division of labor.

Sectoral Diversity is supplied by Harvard University's Atlas of Economic Complexity, and is widely employed in macroeconomic modeling of country-level productivity and economic growth(atlas.cid.harvard.edu; Hausmann et al., 2014). A nation's sectoral diversity score is based on a volume-weighted estimate of its variety of exports - i.e., how many different types of products a nation is able to produce (see Hausmann et al., 2014; Hidalgo \& Hausmann, 2009). Diversity in exports by necessity requires specialization across firms producing goods for export. Sectoral diversity scores therefore reflect division of labor, and thus economic niche diversification, at the country level.

Urbanization and HDI variables are supplied by the United Nations (http://hdr.undp.org/en). Urbanization is the percentage of the nation's population living in urban (vs rural) areas (http://hdr.undp.org/en). The HDI is computed as a nation's average levels of education, gross domestic product per capita, and life expectancy (http://hdr.undp.org/en). Urbanization and HDI reflect a nation's niche diversity, given (1) reciprocal relationships between the division of labor and the degree of urbanization at the national level (Gibbs \& Martin, 1962) and (2) the inter-related effects of the division of labor and urbanization on a nation's per-capita income and wealth. Division of labor and urbanization affect innovation and market productivity and efficiency (e.g., Rodriguez-Clare, 1996; Henrich \& Boyd, 2008), which tends to fuel rising average incomes and standards of living (Hausmann \& Hidalgo et al., 2014; Hidalgo \& Hausmann, 2009). 
Of course, Sectoral Diversity, Urbanization, and HDI are not direct or perfect measures of niche diversity. Each of these components of our niche diversity composite potentially contains variance independent of niche diversity proper that can potentially drive any associations we find, but each also taps different conceptual aspects of niche diversity proper and should contain non-overlapping sources of confounding variance. Importantly, we conducted supplemental analyses to examine the focal relationships using each separate component of the niche diversity index (see Supplemental Materials, Section 3.1), which showed the same pattern of results reported below.

For use in analyses, we pulled estimates of Sectoral Diversity, Urbanization, and HDI for the year 2015, which is the year before the Open-Source Psychometrics began collecting the personality data. Because data were missing on one or more of these indices for several nations, we used a principal components analysis procedure that allows for missing data (Dray \& Dufour, 2007). The first principal component of these three indicators explained $80 \%$ of the variance in the data and loadings on this first principal component were strong for each indicator (HDI $=.80$; Urbanization $=.73$; Sectoral Diversity $=.82$ ). Higher scores on this $z$-scaled index suggest a greater number of specialized niches, whereas lower scores suggest fewer specialized niches within a nation. Figure 1 shows each nation's estimated niche diversity in relation to its sample size. 


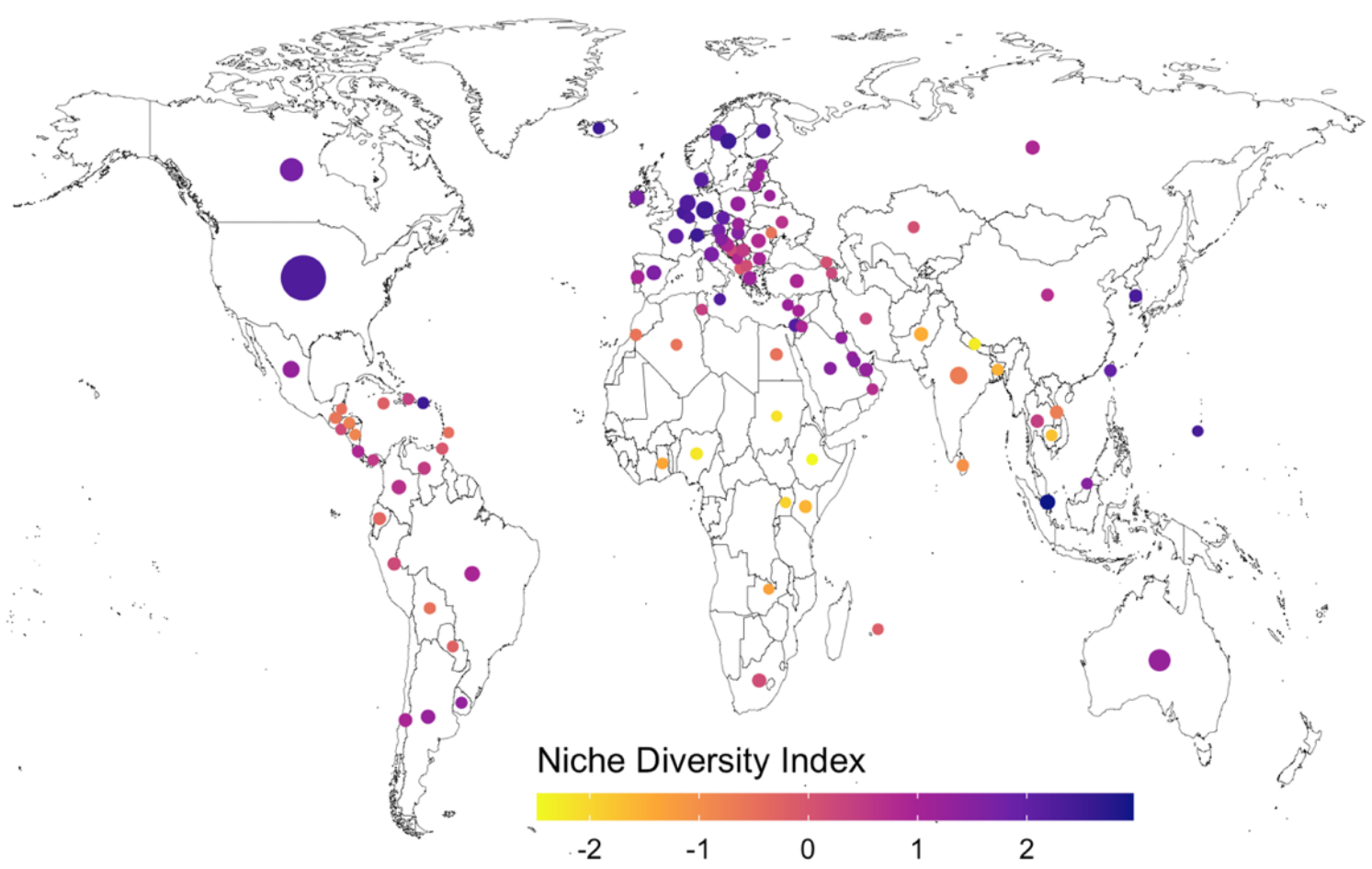

Figure 1. Map highlighting the nations and geographic regions represented by the personality data used in the current study. The centroid size within in each country represents the relative sample size for each nation; nations with smaller points contributed fewer respondents. The hue and luminance of the centroid depicts each nation's standing on the niche diversity index, where nations lower on the niche diversity index are lighter and those higher on the niche diversity index are darker.

\section{Analytic Methods}

We used a two-step analytic procedure. In the first step, we specified the five-factor structure with the IPIP five factor items modeled in the context of a multiple-group model, using the alignment method to maximize approximate measurement invariance across groups (i.e., nations). The alignment allows for meaningful comparison of latent factor means, variances, and covariances across groups without imposing untenable assumptions regarding strict equivalence of factor loadings and intercepts (Asparouhov \& Muthén, 2014). In the second step, we used metaregression to examine the relationship of niche complexity to the covariance and variance parameter estimates extracted from the first step — and their associated standard errors - while 
controlling for several potential confounds. Details of each method are described in their respective sections below.

Multigroup alignment. Our research question requires that we compare variances and covariances across nations. It is therefore necessary to address differences in how the personality items function across nations (Byrne et al., 1989; Church et al., 2011). We addressed measurement invariance using the alignment method (Asparouhov \& Muthén, 2014). Assessments of measurement invariance become intractable with many groups (Marsh et al., 2018). Asparouhov and Muthén (2014) developed the multigroup alignment method as an automated alternative that approximates measurement invariance, allowing for comparison of groups without requiring strict invariance across groups. The multigroup alignment method uses a two-step procedure.

In the first step, the configural model is estimated based on the researcher-specified factor structure where all loadings and intercepts are freely estimated, with factor variances fixed to one and means fixed to zero. Because the alignment method cannot handle cross-loadings or be combined with exploratory structural equation modeling (ESEM) approaches, we assumed the simple Big Five factor structure with no cross-loadings. Typically, omitted cross-loadings will inform the factor covariance estimate, so our factor covariance estimates that we obtain should not be interpreted literally as differences in the relations between "real" dimensions of trait variation, but instead as a more general indication that the constellation of items underlying each set of personality factors differ in their overall magnitude of association with one another. Considering that complex factor structures with many items - like the IPIP-50 used here - are unlikely to meet common fit thresholds (c.f., Marsh et al., 2010), the simple Big Five configural model exhibited reasonable fit in most nations $\left(\right.$ Mean $_{R M S E A}=.07, \operatorname{Min}_{R M S E A}=.05$, Max $_{R M S E A}=$ 
.09; see supplement Section 3.3 for full CFA results in each nation). To examine the degree to which deviations from the simple factor structure may influence our results, we present sensitivity analysis on only nations with acceptable RMSEA values (i.e., below .08) in the results.

In the second step of the alignment procedure, all factor variances and means are iteratively estimated across groups using a loss-simplicity function that minimizes the noninvariance and provides the most invariant pattern across groups. This loss-simplicity function is analogous to different factor rotations in exploratory factor analysis in that the aligned model will have the same fit as the configural model. We used the fixed alignment method, which identifies group factor means and variances in relation to a fixed group whose respective mean and variance remain fixed at zero and one. We opted to set the USA as the reference group in order to facilitate comparison with the broader literature, most of which is based on American participants $^{1}$.

The approximation of measurement invariance provided by the multigroup alignment method is intended to provide more accurate comparison of covariances, variances, and means across groups (Asparouhov \& Muthén, 2014). Our alignment results revealed generally poor invariance across nations (average invariance index $=.33$ ), which is itself consistent with the niche diversity model's prediction that personality factor structure is not uniform across nations. Although this suggests that the alignment method is likely an imperfect solution to the problem of measurement invariance, it still represents an improvement over previous approaches using simple sum scores which do nothing to ameliorate measurement error and bias. Simulations

\footnotetext{
${ }^{1}$ We note that an earlier version of this paper fixed Albania based on the Mplus default settings that fix the first group in the data frame. Importantly, the same pattern of results was obtained in the current version as this earlier version, which is still publicly available (psyarxiv.com/93qmp/).
} 
demonstrate that the alignment method accurately recovers known population parameters even under conditions of substantial non-invariance across items, especially when sample sizes are large, as they generally are in the current sample (Asparouhov \& Muthén, 2014).

Metaregression. The multigroup alignment method does not currently offer support for estimating correlates of group-specific parameters, so examination of the focal associations between niche diversity and the covariance and variance estimates requires additional analytic steps. To examine the focal relationships of niche diversity to personality covariance and variance, we used the metaregression methods described in Tucker-Drob et al. (2019). We constructed separate models for each of the two focal meta-analytic outcomes: (A) nation-level inter-factor covariance estimates and (B) nation-level intra-factor variance estimates. In the interfactor covariance models, the absolute values of each of the ten parameter estimates for the pairwise covariances among Big Five factors across the 115 nations were modelled as the metaanalytic outcome. In the intra-factor variance models, the five parameter estimates for each of the Big Five factor variances across the 115 nations were modelled as the meta-analytic outcome. In all models, we clustered these parameter estimates by nation. We also added precision weights proportional to the inverse sampling variance of the parameter estimates, so that samples with more precise estimates were weighted more strongly in the metaregression analysis.

Effective dimensionality. To examine the focal relationship between niche diversity and the number of emergent personality dimensions in each nation, we implemented the effective dimensionality technique described in Del Giudice (2020). Effective dimensionality provides an estimate of the dimensionality of a set of variables, much like exploratory factor analysis; however, effective dimensionality provides a continuous (i.e., non-discrete) estimate of dimensionality and is agnostic about the underlying causal structure of the variables, making it 
superior to selecting a discrete number of factors based on an arbitrary eigenvalue cutoff. There are several indices of effective dimensionality based on different derivations; we employ the $n_{1}$ index because it does not assign disproportional weight to larger eigenvalues, making it suitable for general purpose estimations of effective dimensionality (for review, see Del Giudice, 2020).

We used the R code provided by Del Giudice (2020) to estimate the effective dimensionality of the Big Five factors in each nation. Importantly, rather than using items as input, we constructed covariance matrices using the inter-factor covariances and the intra-factor variance estimates obtained from the alignment analyses as input for the effective dimensionality analysis, which provides the added benefits of approximate measurement equivalence. We addressed small-sample bias using Mestre's (2008) method (see Del Giudice, 2020 for details).

\section{Analytic Procedure}

We conducted the alignment and metaregression analyses in Mplus (Muthén \& Muthén, 1998-2017). We carried out all other aspects of data cleaning, analyses, and data visualization in R (R Core Team, 2019). All data and code used to conduct the analyses and create the visualizations presented in this paper are provided along with the supplement on the Open Science Framework (https://osf.io/7n4sr).

In our primary metaregression analyses, we regressed each meta-analytic outcome (i.e., Big Five inter-factor covariances and intra-factor variances) on the index of niche diversity. In our primary effective dimensionality analyses, we regressed each nation's effective dimensionality estimate on the index of niche diversity. In secondary analyses, we examined the robustness of the focal associations to alternative explanations and potential confounds by including 11 nation-level controls in our analyses: mortality and homicide rates, acquiescence bias, the Big Five factor means, English proficiency estimates, sample size, and geographic 
region. We describe each control variable and the rationale for its inclusion below. Figure 2 presents the intercorrelations among these nation-level control variables.

Mortality and homicide rates. Međedović (2020) recently proposed that the previously reported differences in personality structure across nations (Lukaszewski et al., 2017; Smaldino et al., 2019) may not be driven by niche diversity, but rather by differences in life history-related behavioral diversification resulting from cross-population variation in exposure to environmental harshness. While "environmental harshness" can be interpreted in multiple ways (Stearns \& Rodrigues, 2020), we included two commonly used nation-level proxies to test against this alternative explanation: homicide rates and mortality rates. We obtained homicide rates from United Nations Office of Drugs and Crime (https://dataunodc.un.org). We log-transformed the homicide rate variable for analyses because it was positively skewed. We computed each nation's mortality rate as the mean of adult male and adult female mortality rates for the year 2015 obtained from The World Bank Group (https://www.worldbank.org). For nations where data were not available for the year 2015, we imputed the estimate from the closest preceding or succeeding year. No mortality estimates were present for any year for Taiwan, so we imputed the mortality rate from neighboring China.

Acquiescence bias. Acquiescence bias refers to the tendency of respondents to be more inclined to agree with items than they are to disagree (or vice versa), potentially confounding variance and covariance estimates. We calculated acquiescence bias according to methods reported in Soto, John, Gosling, \& Potter (2008) by computing the mean for each participant across pairs of items from each IPIP-FFM-50 factor subscales with opposite valence before reverse coding the items. The opposite-valence item pairs from each subscale are: "I don't like to draw attention to myself" and "I don't mind being the center of attention" from the extraversion 
subscale; "I often feel blue" and "I seldom feel blue" from the emotional stability subscale; "I am not really interested in others" and "I am interested in people" from the agreeableness subscale; "I like order" and "I leave my belongings around" from the conscientiousness subscale; and "I do not have a good imagination" and "I have a vivid imagination" from the openness subscale. Higher numbers on the resulting acquiescence score represent tendency to acquiesce ("yeasaying”) whereas lower numbers represent a tendency to dis-acquiesce ("nay-saying”). We averaged the acquiescence score across participants within each nation to estimate nation-level acquiescence scores.

Big Five latent factor means. We included the latent nation means for each of the Big Five factors estimated by the alignment parameter estimates to control for any nation-level differences in personality trait levels that may influence responses to personality scales (e.g., evaluative bias; Lukaszewski et al., 2017), potentially biasing the nation-level trait covariance and variance estimates.

English proficiency. Because the personality survey was only administered in English, a selection bias exists where nations with lower English proficiency were selected against. Respondents from nations with lower English proficiency have a higher potential to misunderstand the questions, creating bias. To assess whether English proficiency could be driving any observed effects, we conducted a sensitivity analysis using just the nations where English is an official language (either de facto or de jure official).

We also included nation-level estimates of English proficiency provided by Education First (https://www.ef.edu) as a control in our robustness analyses. For nations where there were no English proficiency data from the year 2015, we imputed the closest available preceding or succeeding estimate. English proficiency data were completely unavailable for 27 nations, so we 
imputed either A) the highest available English proficiency estimate for the 21 nations where proficiency data were missing but English is an official language, or B) the mean English proficiency estimate for six nations where English is not an official language and proficiency data were missing.

Sample size. Lukaszewski et al. (2017) included sample size as a control to demonstrate the differences in accuracy of covariance estimates were not driving the relationship to niche diversity. Our metaregression analyses with inverse variance weights already account for the influence of sample size on the covariance and variance estimates and our effective dimensionality also correct for sample size bias. Still, we include sample size as a control variable in robustness analyses because the sample size contributed by each nation likely proxies for potentially unmeasured confounds related to differential selection. We log-transformed this sample size variable because it was positively skewed.

Geographic region. We included dummy-coded variables representing the continent each nation is located on to control for non-independence of observations due to geospatial proximity. 


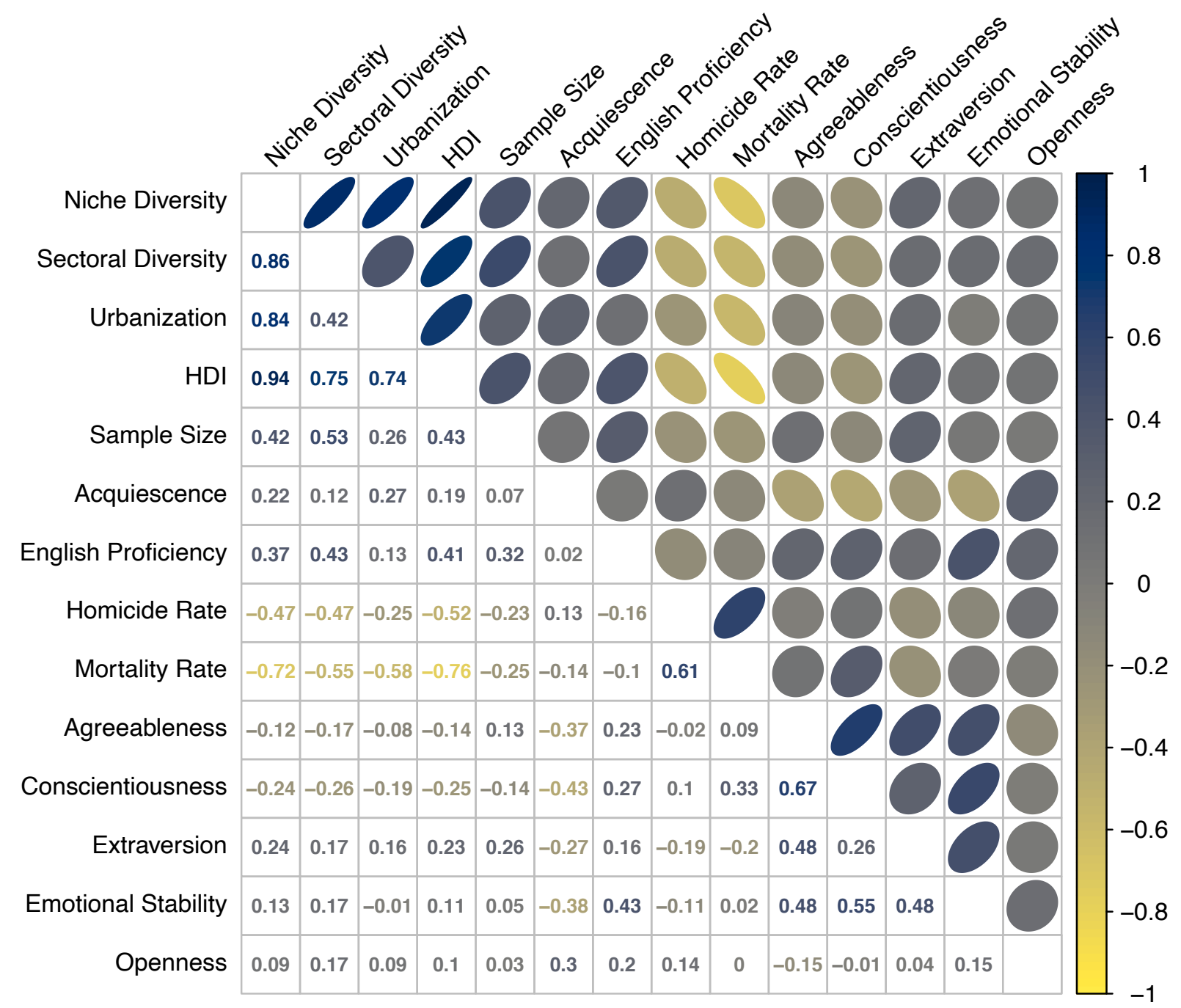

Figure 2. Intercorrelations among nation-level niche diversity estimates and nation-level covariates. The personality controls labelled for each Big Five factor refer to the alignment-estimated latent factor means for each nation. Nation-level acquiescence is calculated as the mean acquiescence score across respondents within each nation. Sample size and homicide rate are log-transformed.

\section{Results}

\section{Primary analyses}

Across nations, the mean inter-factor covariance estimate was .18 $(S D=.02)$, the mean intra-factor variance estimate was $.86(S D=.03)$, and the mean effective dimensionality estimate was $4.47(S D=.32)$. Inter-factor covariance estimates were positively correlated with intra- 
factor variance estimates $(r=.52)$ and negatively correlated with effective dimensionality estimates $(r=-.78)$. Effective dimensionality estimates were negatively correlated with intrafactor variance estimates $(r=-.35)$.

Figure 3 shows the results of the primary metaregression analyses examining overall inter-factor covariance, intra-factor variance, and effective dimensionality as a function of niche diversity $^{2}$. Each standard deviation increase in niche diversity was associated with .52 standard deviations lower covariance among the Big Five factors; in terms of raw units, each standard deviation increase in niche diversity was associated with .02 lower inter-factor covariance. The association between niche diversity and variance within the Big Five factors was not statistically different from zero. Finally, each standard deviation increase in niche diversity was associated with a .53 standard deviation increase in effective dimensionality, or in raw units, each standard deviation increase in niche diversity was associated with an average of .13 more effective dimensions of personality. This pattern of results is obtained using each of the individual predictors of niche diversity as well (see Supplemental Materials, Section 3.1).

\footnotetext{
${ }^{2}$ For readers interested in attempting to disentangle the degree to which differences between the current results and the results of previous research reflect the different statistical approach versus the increased sample size, we report results of additional comparative analyses that rely on covariance and variance estimates across simple factor meanscores, rather than alignment-based factor estimates, in the OSF Supplement Section 3 (https://osf.io/7n4sr/). To briefly summarize: inter-factor covariance and effective dimensionality are both slightly more strongly associated with niche diversity under the alignment-based approach than the factor mean-score approach. However, the association between niche diversity and intra-factor variance is slightly weaker under the alignment-based approach than the mean-score approach (but not robust against controls in either approach).
} 

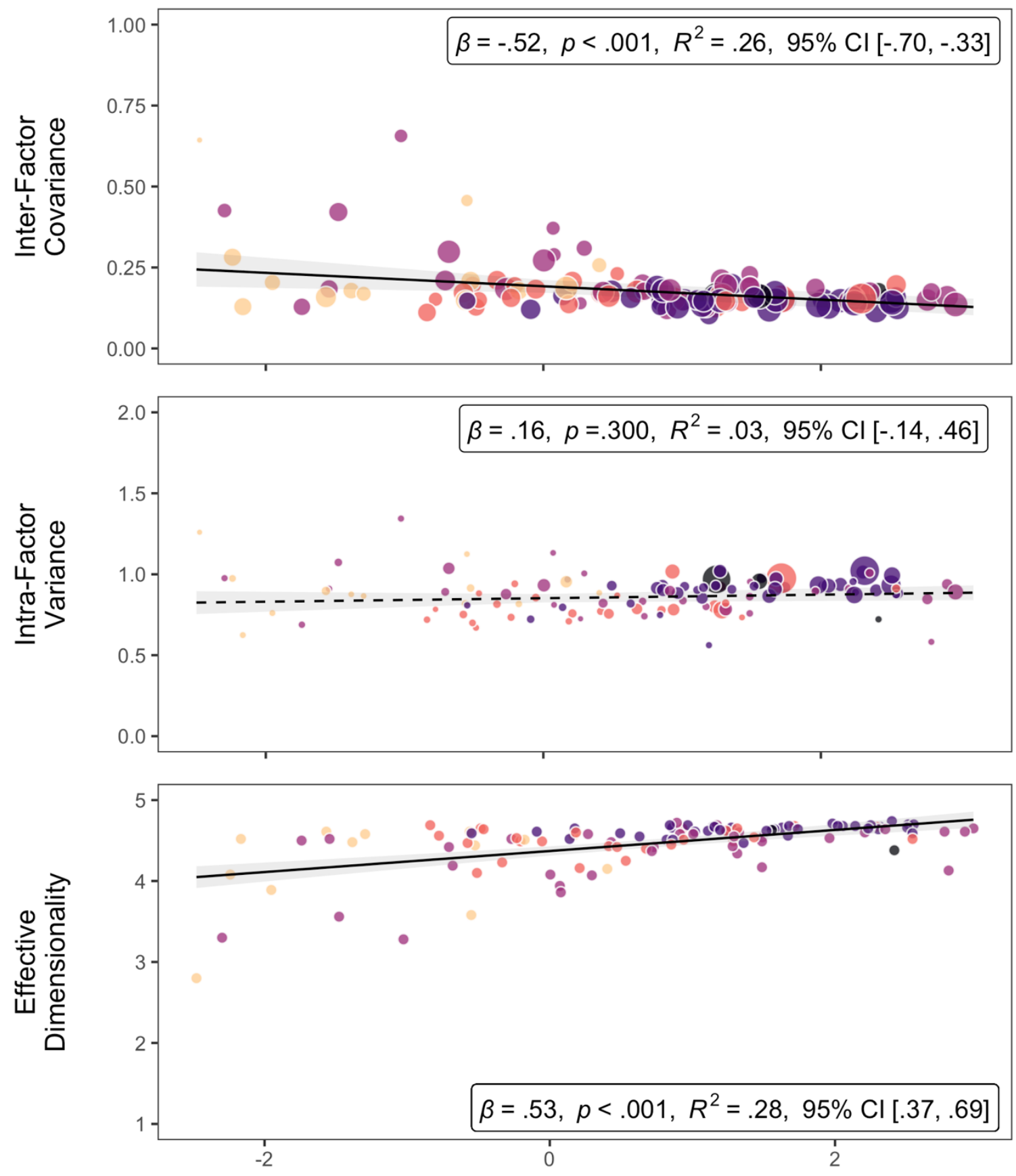

Niche Diversity Index

\section{Africa - Americas - Asia - Europe - Oceania}

Figure 3. Bubble plot depicting (1) absolute overall inter-factor covariances, (2) overall intra-factor variances, and (3) effective dimensionality of the Big Five traits across 115 nations as a function of nations' standing on the niche diversity index. The size of each bubble in the intra-factor variance and inter-factor covariance plots are proportional to the inverse sampling variance weight given to the estimates for each country, where larger circles indicate greater weight in the analysis resulting from smaller standard errors and less sampling variance. 
Sensitivity checks. Because the use of absolute values of the covariance estimates in our analyses may bias estimates, we also conducted analyses in which we did not force all estimates to take on positive values. We reversed the covariance estimates for pairs that were on average negative and left any negative estimates untouched for pairs where the mean covariance was positive. Thus, for any given pair of variables, covariances could take on both positive and negative values. Meta-regression results were not appreciably different from the analyses using absolute values of the covariance estimates $(\beta=-.48, p=.001,95 \% \mathrm{CI}[-.74,-.21])$. To disentangle any potential confounding effects of personality variance on personality covariance, we conducted analyses of inter-factor correlations, rather than covariances, by scaling the interfactor covariances and their standard errors relative to their respective intra-factor variances. The association with niche diversity was slightly stronger than when using covariances $(\beta=-.69, p<$ $.001,95 \%$ CI $[-.92,-.46])$.

There was more variability in the residual inter-factor covariance and effective dimensionality estimates at low levels of niche diversity than at high levels. We therefore examined the focal relationships in only the 84 countries with niche diversity scores greater than zero, for which the residuals were more homoscedastic. The negative inter-factor covariance association $(\beta=-.41, p<.001,95 \% \mathrm{CI}[-.61,-.21])$ and the positive effective dimensionality associations $(\beta=.43, p<.001,95 \%$ CI $[.24, .61])$ were both robust to these sensitivity checks. Restricting our analyses to the 42 nations in the sample with English as an official language, did not substantively change the associations between niche diversity and inter-factor covariance $(\beta$ $=-.41, p=.003,95 \%$ CI [-.68, -.14]), intra-factor variance $(\beta=.01, p=.961,95 \% \mathrm{CI}[-.41, .43])$, or effective dimensionality $(\beta=.52, p<.001,95 \%$ CI $[.24, .79])$. Finally, in analyses including only the 90 nations that exhibited reasonable fit in CFAs (i.e., RMSEA <.08), the associations 
are qualitatively unchanged between niche diversity and inter-factor covariances $(\beta=-.51, p<$ $.001,95 \% \mathrm{CI}[-.69,-.32])$, intra-factor variances $(\beta=.18, p=.100,95 \% \mathrm{CI}[-.03, .39])$, and effective dimensionality $(\beta=.43, p<.001,95 \%$ CI $[.29, .56])$.

\section{Robustness analyses}

We conducted secondary metaregression analyses to examine the robustness of the focal relationships to potential confounds. We added the sample size for each nation, nation-level estimates of acquiescence bias, alignment-estimated latent factor means for each of the Big Five traits for each nation (i.e., evaluative bias), English proficiency, homicide rate, mortality rate, and dummy-codes representing the macro-geographic regions as controls for geospatial dependence to the primary models. Inspection of the Variance Inflation Factors for covariates indicated that multicollinearity was not problematic for parameter estimation (range: 1.28-5.35). Figure 4 shows the results of the robustness tests and we provide tabular output with exact $p$ values for all parameters in the supplementary materials (Section 3.1).

As shown in Figure 4, when all controls were included as predictors, niche diversity was still negatively associated with Big Five inter-factor covariances $(\beta=-.52, p<.001,95 \%$ CI [$.75,-.28])$ and positively associated with effective dimensionality $(\beta=.41, p<.001,95 \% \mathrm{CI}$ $[.20, .62])$. The association between niche diversity and intra-factor variance became negative with the inclusion of controls but remained non-significant $(\beta=-.25, p=.095,95 \% \mathrm{CI}[-.55$, .04]). Nation-level acquiescence bias and conscientiousness were significantly negatively associated with inter-factor covariances and significantly positively associated with effective dimensionality across nations (see Figure 4 for effect sizes and confidence intervals). Nationlevel extraversion was also significantly negatively associated with effective dimensionality and significantly positively associated with inter-factor covariance (see Figure 4 for effect sizes and 
confidence intervals). Intra-factor variance across nations was significantly positively associated with nations' sample size and average openness, and significantly negatively related to nations' acquiescence bias (see Figure 4 for effect sizes and confidence intervals). We also examined the effects of the different sets of control variables by conducting separate analyses entering the controls in a step-wise manner; the details of these results are provided in the Supplemental Materials (Section 3.1).

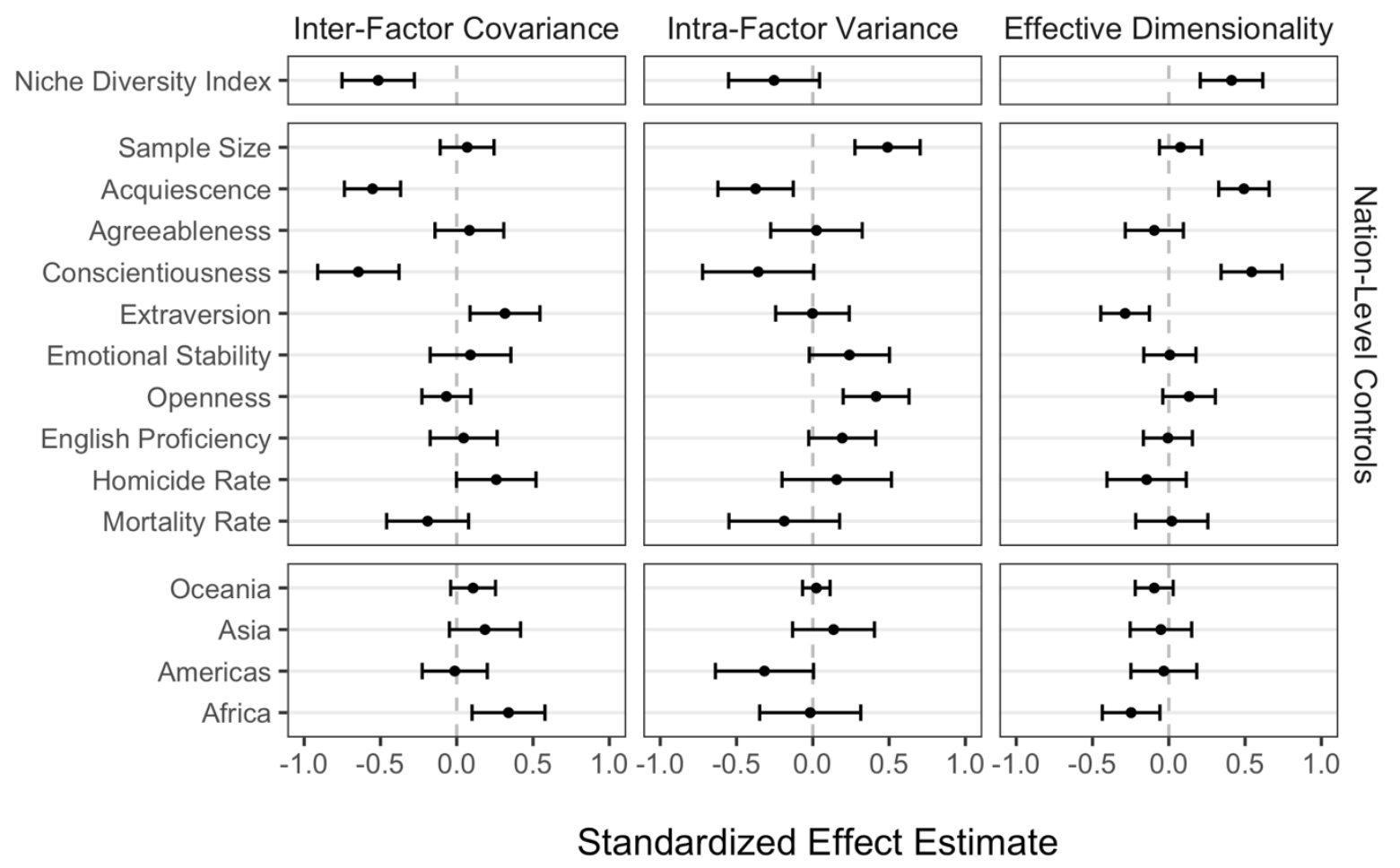

Figure 4. Point estimates and $95 \%$ confidence intervals for focal associations with overall absolute interfactor covariance, overall intra-factor variance, and effective dimensionality after including controls, along with point estimates and $95 \%$ confidence intervals for the control variables. The reference group for the continent dummy-variable is Europe. The personality controls labelled for each Big Five factor refer to the alignment-estimated latent factor means for each nation. Sample size and homicide rate are log-transformed.

\section{Exploratory analyses of pairwise covariance and individual variances}

Figure 5 shows the associations (with and without controls) between niche diversity and (A) each of the 10 pairwise absolute covariance estimates and (B) each individual intra-factor 
variance estimate. One of the ten pairs of Big Five inter-factor covariances were significantly associated with niche diversity in the same direction in both the controlled and uncontrolled analyses: conscientiousness with extraversion. No other pairwise factor covariances were significantly associated with niche diversity in the same direction both with and without controls. None of the associations between niche diversity and individual Big Five intra-factor variances were statistically significant and in the same direction in analyses with and without controls.

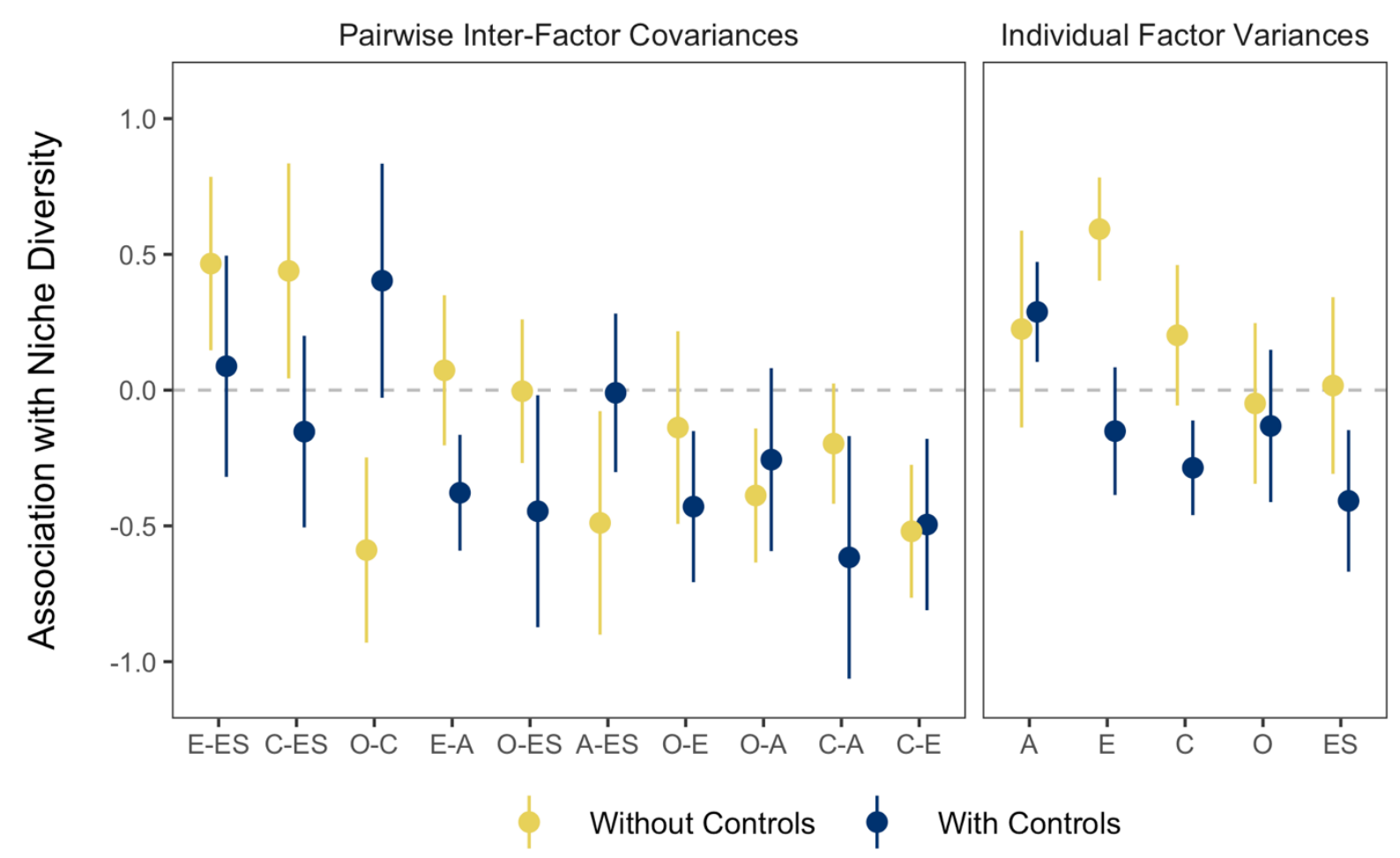

Figure 5. Point estimates and $95 \%$ confidence intervals for the associations between niche diversity and (a) the absolute inter-factor covariances among all possible pairs of Big Five traits; and (b) each of the individual Big Five intra-factor variances. The light-colored point estimates and $95 \% \mathrm{Cls}$ are without the control variables and dark-colored point estimates and $95 \% \mathrm{Cls}$ are after controlling for the 11 control variables. $\mathrm{A}=$ Agreeableness; $\mathrm{C}=$ Conscientiousness; $\mathrm{E}=$ Extraversion; $\mathrm{O}=$ Openness; $\mathrm{ES}=$ Emotional Stability.

\section{Discussion}

The niche diversity hypothesis proposes that personality structure is a function of the number of socioecological niches within a population (Lukaszewski et al., 2017; Smaldino et al., 2019). It predicts that increasing niche diversity is associated with (i) lower personality trait 
covariance; (ii) greater personality trait variance; and (iii) greater personality dimensionality. We improved upon the only prior empirical test of this hypothesis by more than doubling the number of nations under investigation and implementing methods to mitigate against confounds associated with differences in the measurement properties of personality scales across nations. In a sample of 685,089 individuals across 115 nations, we found robust evidence for the first and third predictions, but little overall support for the second.

As predicted, we found that personality traits were less distinct in nations with lower niche diversity. Not only was this association obtained using a psychometric method to minimize artifacts associated with measurement inequivalence across nations, but the association remained after statistically controlling for several potential confounds. Importantly, niche diversity explained variation in personality structure in the presence of environmental-harshness indicators, which casts doubt on an alternative explanation proposed by Međedović (2020) that population differences in personality structure may be accounted for by the effects of environmental harshness on behavioral diversification along the life history spectrum.

We did not find robust support for the model-predicted relationship between niche diversity and overall trait variance. This association was not statistically significant and reversed when controlling for potential confounds. This finding indicate that the association between trait variance and niche diversity within human populations may not be as straightforward as prior simulations and data suggested (Smaldino et al., 2019) and reveal where further refinement of the hypothesis may be fruitful. Perhaps, niche diversity does not inevitably lead to greater trait variance if many niches within and between populations tend to incentivize similar levels of traits when they are incentivized at all. If this were the case, the diversity of niche-incentivized 
trait combinations could still vary across populations, leading to higher personality dimensionality and less overall covariance among traits, as we found.

One limitation of this study is that the personality survey was administered only in English via the internet. This enabled us to use identical personality measures across all participants and nations, avoiding confounds associated with translation. It does mean, however, that many of the participants responded to a survey in their non-native language and that individuals who were not English-literate were unable to participate. We took several steps to guard against this potential language confound. First, our implementation of the alignment method allowed us to approximate measurement invariance across nations. Second, our metaregression models adjusted for variation in acquiescent responding across nations and variation in national estimates of English proficiency. Importantly, selection bias associated with both English literacy and internet access may actually render our results more conservative if participants tended to come from more urban, niche-diverse regions of their countries. Nonetheless, personality assessments administered in respondents' preferred languages, allowing more representative sampling, is necessary to fully assess the generalizability of our findings.

Another limitation is that our data lack demographic information about participants. Prior research suggests that the factor structure of personality may vary with age (Beck et al., 2019; Mottus et al., 2019; Soto et al., 2008). If the age distribution of participants within each nation systematically varied with niche diversity, then the results presented here could be confounded with age trends in the personality structure. Although we do not have a strong reason to suspect such confounding, the lack demographic data prevents us from exploring this issue here. We speculate here that the niche diversity hypothesis might partly explain age trends in personality structure. For instance, observed age differences in personality structure among US participants 
(Beck et al., 2019) appear to track trends of workforce participation and thereby occupational niche diversification. Examining the extent to which age-related differences in personality structure coincide with age differences in niche diversity is an interesting future direction.

Finally, our analysis of personality structure relied on the Big Five factor structure even though the niche diversity hypothesis explicitly presumes the structure of personality to vary across cultures. Because it was not possible to combine the alignment with ESEM approaches, we chose alignment to prioritize maximizing the comparability of personality traits across countries. To quote Cronbach and Meehl (1995), we use the simple five-factor factor model as a tool for "defining a working reference frame, located in a convenient manner," rather than to discern "'real dimensions' [in which] a great deal of surplus meaning is implied" (p. 277-278). Investigating the degree to which complex factor solutions and other personality frameworks yield different patterns of association with niche diversity is a key future direction.

The niche diversity hypothesis was partly motivated by recognizing that personality science could benefit from model-building from first principles. Our findings provide the strongest support to date for the central empirical predictions generated by the niche diversity hypothesis, demonstrating the contingency of personality structure on socioecological dynamics. Further considerations of the socioecological factors that vary within and between human societies will be crucial for refining our understanding of the nature of personality and psychological variation more generally. 


\section{References}

Allik, J., \& McCrae, R. R. (2004). Toward a geography of personality traits: Patterns of profiles across 36 cultures. Journal of Cross-Cultural Psychology, 35(1), 13-28.

Asparouhov, T., \& Muthén, B. (2014). Multiple-group factor analysis alignment. Structural Equation Modeling: A Multidisciplinary Journal, 21(4), 495-508.

Beck, E. D., Condon, D. M., \& Jackson, J. J. (2019, July 9). Interindividual Age Differences in Personality Structure. https://doi.org/10.31234/osf.io/857ev

Briley, D. A., \& Tucker-Drob, E. M. (2014). Genetic and environmental continuity in personality development: A meta-analysis. Psychological bulletin, 140(5), 1303.

Briley, D. A., \& Tucker-Drob, E. M. (2017). Comparing the developmental genetics of cognition and personality over the life span. Journal of Personality, 85(1), 51-64.

Byrne, B. M., Shavelson, R. J., \& Muthén, B. (1989). Testing for the equivalence of factor covariance and mean structures: the issue of partial measurement invariance. Psychological bulletin, 105(3), 456.

Chen, Y., Culpepper, S., \& Liang, F. (2020). A Sparse Latent Class Model for Cognitive Diagnosis. Psychometrika, 1-33.

Church, A. T., Alvarez, J. M., Mai, N. T., French, B. F., Katigbak, M. S., \& Ortiz, F. A. (2011). Are cross-cultural comparisons of personality profiles meaningful? Differential item and facet functioning in the Revised NEO Personality Inventory. Journal of personality and social psychology, 101(5), 1068.

Cronbach, L. J., \& Meehl, P. E. (1955). Construct validity in psychological tests. Psychological bulletin, 52(4), 281. 
Del Giudice, M. (2020). Effective Dimensionality: A Tutorial. Multivariate Behavioral Research, 1-16.

Denissen, J. J., Bleidorn, W., Hennecke, M., Luhmann, M., Orth, U., Specht, J., \& Zimmermann, J. (2018). Uncovering the power of personality to shape income. Psychological science, 29(1), 3-13.

Digman, J. M. (1990). Personality structure: Emergence of the five-factor model. Annual review of psychology, 41(1), 417-440.

Digman, J. M. (1997). Higher-order factors of the Big Five. Journal of personality and social psychology, 73(6), 1246.

Dray, S., \& Dufour, A. B. (2007). The ade4 package: implementing the duality diagram for ecologists. Journal of statistical software, 22(4), 1-20.

Ehrhart, K. H., Roesch, S. C., Ehrhart, M. G., \& Kilian, B. (2008). A test of the factor structure equivalence of the 50-item IPIP Five-factor model measure across gender and ethnic groups. Journal of Personality Assessment, 90(5), 507-516.

Gibbs, J. P., \& Martin, W. T. (1962). Urbanization, technology, and the division of labor: International patterns. American sociological review, 667-677.

Goldberg, L. R. (1992). The development of markers for the Big-Five factor structure. Psychological assessment, 4(1), 26.

Gurven, M., Von Rueden, C., Massenkoff, M., Kaplan, H., \& Lero Vie, M. (2013). How universal is the Big Five? Testing the five-factor model of personality variation among forager-farmers in the Bolivian Amazon. Journal of personality and social psychology, 104(2), 354. 
Hausmann, R., Hidalgo, C. A., Bustos, S., Coscia, M., \& Simoes, A. (2014). The atlas of economic complexity: Mapping paths to prosperity. Mit Press.

Heine, S. J., \& Buchtel, E. E. (2009). Personality: The universal and the culturally specific. Annual review of psychology, 60, 369-394.

Henrich, J. (2015). Culture and social behavior. Current Opinion in Behavioral Sciences, 3, 8489.

Henrich, J., \& Boyd, R. (2008). Division of labor, economic specialization, and the evolution of social stratification. Current Anthropology, 49(4), 715-724.

Hidalgo, C. A., \& Hausmann, R. (2009). The building blocks of economic complexity. Proceedings of the national academy of sciences, 106(26), 10570-10575.

Hirschfeld, G., von Brachel, R., \& Thielsch, M. (2014). Selecting items for Big Five questionnaires: At what sample size do factor loadings stabilize?. Journal of Research in Personality, 53, 54-63.

Kaiser, T., Del Giudice, M., \& Booth, T. (2019). Global sex differences in personality: Replication with an open online dataset. Journal of personality.

Kajonius, P., \& Mac Giolla, E. (2017). Personality traits across countries: Support for similarities rather than differences. PloS one, 12(6).

Kaplan, H. S., Hooper, P. L., \& Gurven, M. (2009). The evolutionary and ecological roots of human social organization. Philosophical Transactions of the Royal Society B: Biological Sciences, 364(1533), 3289-3299.

Lee, K., \& Ashton, M. C. (2004). Psychometric properties of the HEXACO personality inventory. Multivariate behavioral research, 39(2), 329-358. 
Legare, C. H. (2017). Cumulative cultural learning: Development and diversity. Proceedings of the National Academy of Sciences, 114(30), 7877-7883.

Lim, B. C., \& Ployhart, R. E. (2006). Assessing the convergent and discriminant validity of Goldberg's International Personality Item Pool: A multitrait-multimethod examination. Organizational Research Methods, 9, 29-54.

Lukaszewski, A. W., Gurven, M., von Rueden, C. R., \& Schmitt, D. P. (2017). What explains personality covariation? A test of the niche diversity hypothesis. Social Psychological and Personality Science, 8(8), 943-952.

Marsh, H. W., Guo, J., Parker, P. D., Nagengast, B., Asparouhov, T., Muthén, B., \& Dicke, T. (2018). What to do when scalar invariance fails: The extended alignment method for multi-group factor analysis comparison of latent means across many groups. Psychological Methods, 23(3), 524.

McCrae, R. R., \& Costa Jr, P. T. (1997). Personality trait structure as a human universal. American psychologist, 52(5), 509.

McCrae, R. R., \& Costa Jr, P. T. (2008). Empirical and theoretical status of the five-factor model of personality traits.

McCrae, R. R., Costa Jr, P. T., Del Pilar, G. H., Rolland, J. P., \& Parker, W. D. (1998). Crosscultural assessment of the five-factor model: The Revised NEO Personality Inventory. Journal of Cross-Cultural Psychology, 29(1), 171-188.

McCrae, R. R., \& Terracciano, A. (2005). Personality profiles of cultures: aggregate personality traits. Journal of personality and social psychology, 89(3), 407. 
Međedović, J. (2020). An Evolutionary Ecological Framework for Understanding Human Behavioral Syndromes: Commentary on Lukaszewski et al. 2017. Social Psychological and Personality Science, 11(4), 570-573.

Mestre, X. (2008). Improved estimation of eigenvalues and eigenvectors of covariance matrices using their sample estimates. IEEE Transactions on Information Theory, 54, 5113-5129. doi:10.1109/TIT.2008.929938

Mõttus, R., Briley, D. A., Zheng, A., Mann, F. D., Engelhardt, L. E., Tackett, J. L., ... \& TuckerDrob, E. M. (2019). Kids becoming less alike: A behavioral genetic analysis of developmental increases in personality variance from childhood to adolescence. Journal of personality and social psychology, 117(3), 635.

Muthén, L. K., \& Muthén, B. O. (1998-2017). Mplus user's guide (8th ed.). Los Angeles, CA: Author.

Nettle, D. (2009). Personality: What makes you the way you are. Oxford University Press.

Pinker, S. (2010). The cognitive niche: Coevolution of intelligence, sociality, and language. Proceedings of the National Academy of Sciences, 107(2), 8993-8999.

R Core Team (2019). R: A language and environment for statistical computing. R Foundation for Statistical Computing, Vienna, Austria. URL: https://www.R-project.org/.

Rodriguez-Clare, A. (1996). The division of labor and economic development. Journal of Development Economics, 49(1), 3-32.

Saucier, G., \& Goldberg, L. R. (1996). Evidence for the Big Five in analyses of familiar English personality adjectives. European Journal of Personality, 10(1), 61-77. 
Saucier, G., Thalmayer, A. G., Payne, D. L., Carlson, R., Sanogo, L., Ole-Kotikash, L., ... \& Szirmák, Z. (2014). A basic bivariate structure of personality attributes evident across nine languages. Journal of Personality, 82(1), 1-14.

Sih, A., Mathot, K. J., Moirón, M., Montiglio, P. O., Wolf, M., \& Dingemanse, N. J. (2015). Animal personality and state-behaviour feedbacks: a review and guide for empiricists. Trends in ecology \& evolution, 30(1), 50-60.

Schmitt, D. P., Allik, J., McCrae, R. R., \& Benet-Martínez, V. (2007). The geographic distribution of Big Five personality traits: Patterns and profiles of human self-description across 56 nations. Journal of cross-cultural psychology, 38(2), 173-212.

Smaldino, P. E., Lukaszewski, A., von Rueden, C., \& Gurven, M. (2019). Niche diversity can explain cross-cultural differences in personality structure. Nature Human Behaviour, 3(12), 1276-1283.

Soto, C. J., John, O. P., Gosling, S. D., \& Potter, J. (2008). The developmental psychometrics of big five self-reports: Acquiescence, factor structure, coherence, and differentiation from ages 10 to 20. Journal of personality and social psychology, 94(4), 718.

Terracciano, A., McCrae, R. R., \& Costa Jr, P. T. (2010). Intra-individual change in personality stability and age. Journal of research in personality, 44(1), 31-37.

Tooby, J., \& DeVore, I. (1987). The reconstruction of hominid behavioral evolution through strategic modeling. In W. Kinzey (Ed.), Primate Models of Hominid Behavior. New York: SUNY Press.

Tucker-Drob, E. M., Brandmaier, A. M., \& Lindenberger, U. (2019). Coupled cognitive changes in adulthood: A meta-analysis. Psychological bulletin, 145(3), 273-301. 